\title{
Noninvasive cardiac output measurement using the volume clamp method in cardiosurgical intensive care unit patients: A comparison with the pulmonary artery catheter
}

\author{
Julia Y Wagner ${ }^{1}$; Annmarie Körner ${ }^{1}$; Mathias Kubik ${ }^{2,3}$; Stefan Kluge ${ }^{3}$; Daniel A Reuter ${ }^{1}$; Bernd Saugel ${ }^{1}$
}

\begin{abstract}
${ }^{1}$ Department of Anesthesiology, Center of Anesthesiology and Intensive Care Medicine, ${ }^{2}$ Department of Cardiovascular Surgery, University Heart Center Hamburg, ${ }^{3}$ Department of Intensive Care Medicine, University Medical Center Hamburg-Eppendorf, Martinistrasse 52, 20246 Hamburg, Germany
\end{abstract}

\begin{abstract}
Introduction: The CNAP technology (CNSystems Medizintechnik AG, Graz, Austria) provides noninvasive continuous recording of the arterial pressure waveform based on the volume clamp method (Fig. 1). Recently, an algorithm for measuring cardiac output (CO) using pulse contour analysis of the CNAP-derived arterial waveform became available. In this study, we compared $\mathrm{CO}$ measurements and trending capability of the novel CNAP-CO (CNCO) with intermittent invasive $\mathrm{CO}$ measurements derived from the pulmonary artery catheter (PAC; PAC-CO) in cardiosurgical intensive care unit patients.
\end{abstract}

Methods: In this interim analysis, we analyzed simultaneously obtained $\mathrm{CNCO}$ and PAC-CO measurements in 41 patients during the first hours after off pump coronary artery bypass surgery. We performed 3 independent sets of 5 consecutive thermodilution measurements each per patient. The average of the 3 closest of the 5 PAC-CO measurements was used for comparison with the average of the corresponding $\mathrm{CNCO}$ values.

Four pairs of measurements were excluded due to artifacts resulting in 119 paired measurements for analysis. In addition, we analyzed 27 cardiac output-modifying maneuvers to evaluate trending ability. We conducted 2 separate comparative analyses: 1) CNCO calibrated to the first simultaneously measured PAC-CO value $\left(\mathrm{CNCO}_{\text {cal }}\right)$ vs. PAC-CO and 2) $\mathrm{CNCO}$ auto-calibrated to biometric patient data $\left(\mathrm{CNCO}_{\text {bio }}\right)$ vs. PAC-CO.

Agreement between the two methods was statistically assessed by Bland-Altman analysis and by calculating the percentage error (PE). For evaluating trending ability, we calculated the concordance rate (CCR; exclusion zone $0.5 \mathrm{~L} / \mathrm{min}$ ).

Results: For $\mathrm{CNCO}_{\text {cal }}$, the Bland-Altman analysis revealed a mean difference of $-0.2 \mathrm{~L} / \mathrm{min}$, a standard deviation of $\pm 0.5 \mathrm{~L} / \mathrm{min}$ and limits of agreement of -1.1 to $+0.8 \mathrm{~L} / \mathrm{min}$ (Fig. 2). The PE and CCR were $19 \%$ and $100 \%$, respectively. For $\mathrm{CNCO}_{\text {bio }}$, the Bland-Altman analysis showed a mean difference of $+0.6 \mathrm{~L} / \mathrm{min}$, a standard deviation of $\pm 1.1 \mathrm{~L} /$ min and limits of agreement of -1.6 to $+2.8 \mathrm{~L} / \mathrm{min}$. The PE and CCR were $45 \%$ and $94 \%$, respectively.

Conclusion: In this clinical study in cardiosurgical intensive care unit patients, $\mathrm{CNCO}_{\text {cal }}$ showed good agreement (PE 19\%) and good trending capability (CCR 100\%) when compared with intermittent pulmonary artery thermodilution. For $\mathrm{CNCO}_{\text {bio }}$, we observed a higher PE (45\%) but acceptable trending capability (CCR 94\%).

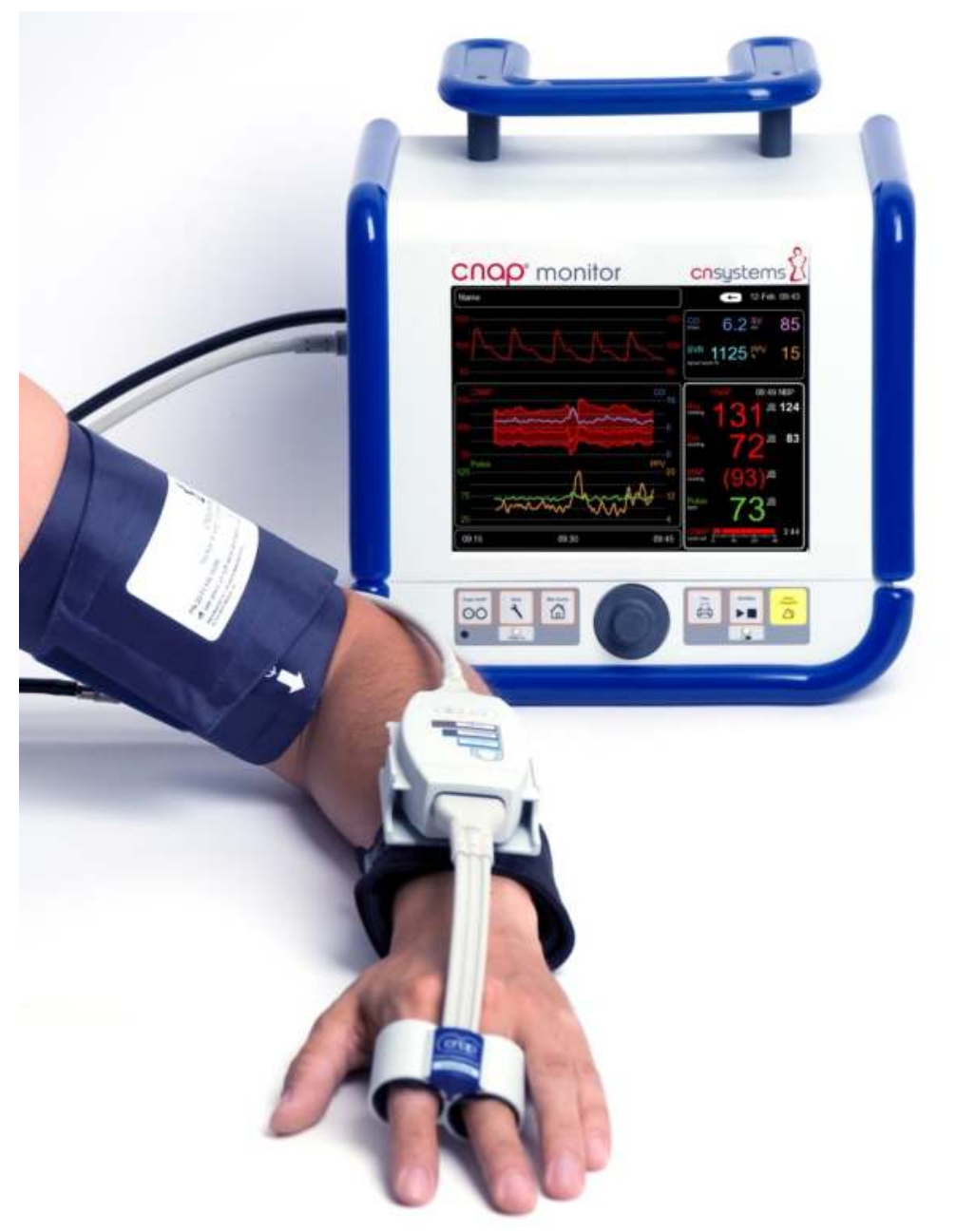

Fig. 1. The CNAP technology (source: CNSystems Medizintechnik AG, Graz, Austria)

Conflicts of interest:
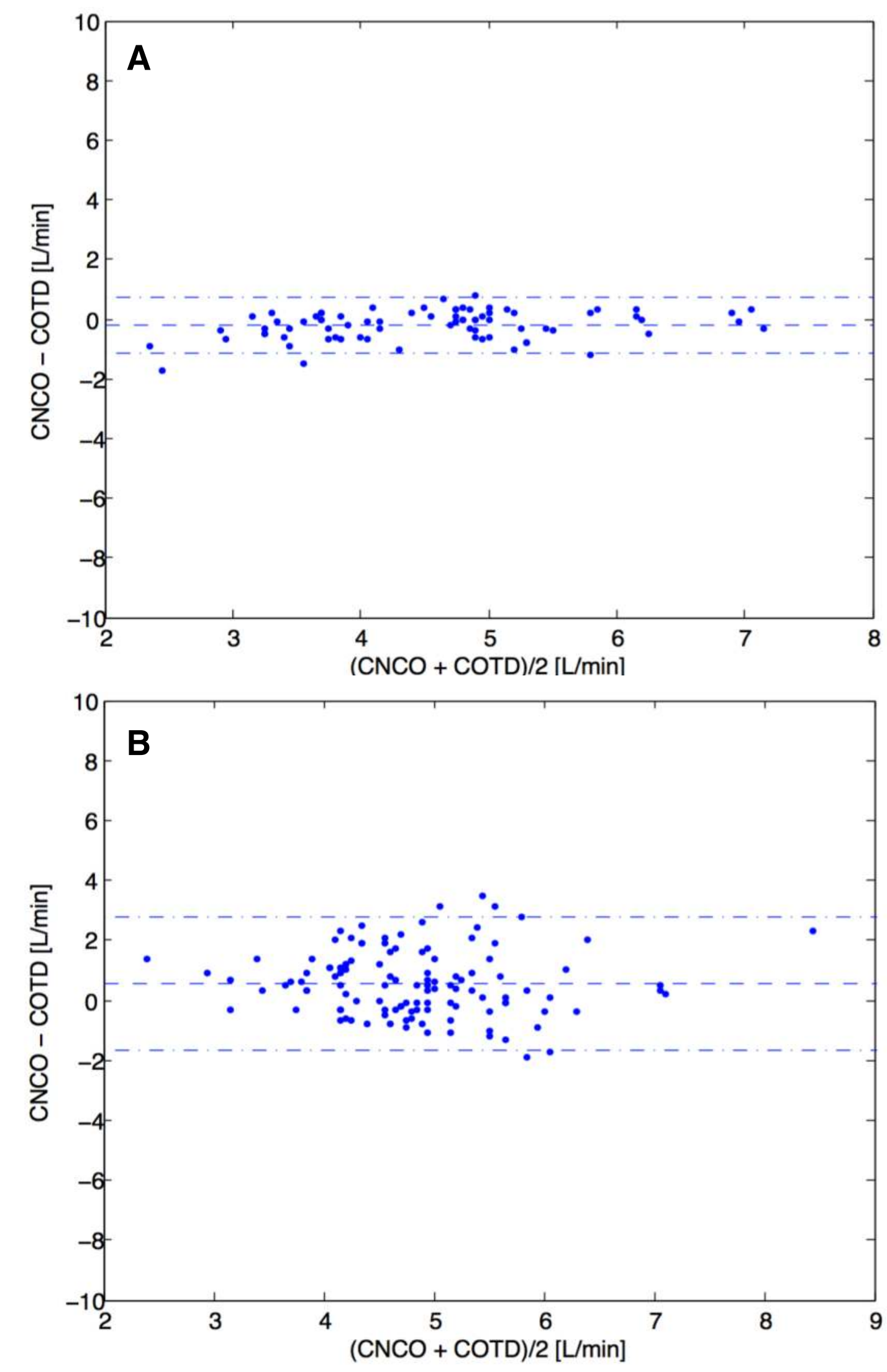

Fig. 2. Bland-Altman analysis for CNCO calibrated to the first simultaneously measured $P A C-C O$ value $(A)$ and auto-calibrated to biometric patient data $(B)$ in 41 patients.

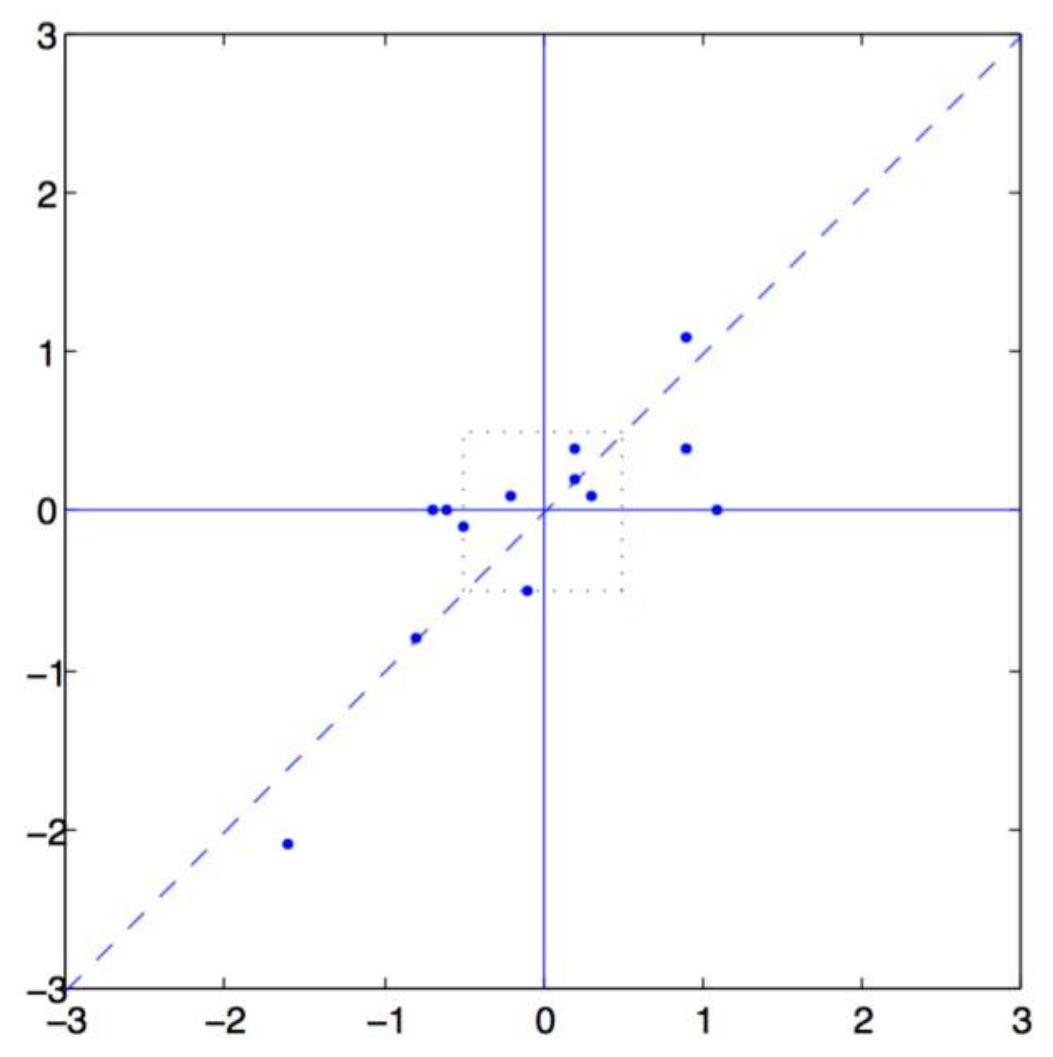

Fig. 3. Fourquadrant plot with a $0.5 \mathrm{~L} / \mathrm{min}$ exclusion zone.

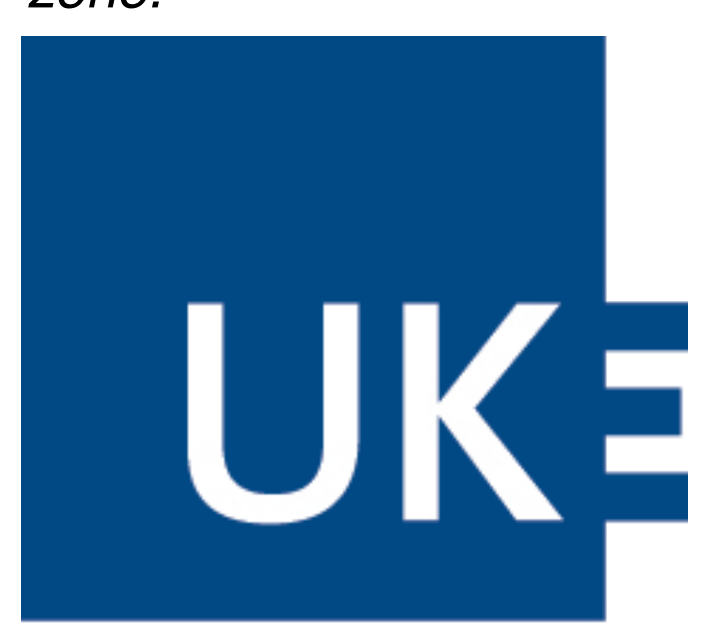

JYW and BS received refunds of travel expenses and $B S$ received lecture $H A M B \cup R G$ fees from CNSystems Medizintechnik AG (Graz, Austria). For all other authors there is no conflict of interest to disclose 